\title{
Point-Light Biological Motion Perception Activates Human Premotor Cortex
}

\author{
Ayse Pinar Saygin, ${ }^{1}$ Stephen M. Wilson, ${ }^{2}$ Donald J. Hagler Jr, ${ }^{1}$ Elizabeth Bates,${ }^{1 \dagger}$ and Martin I. Sereno ${ }^{1}$ \\ ${ }^{1}$ Department of Cognitive Science, University of California, San Diego, La Jolla, California 92093-0515, and 2Ahmanson-Lovelace Brain Mapping Center and \\ Neuroscience Interdepartmental Program, University of California, Los Angeles, California 90095
}

\begin{abstract}
Motion cues can be surprisingly powerful in defining objects and events. Specifically, a handful of point-lights attached to the joints of a human actor will evoke a vivid percept of action when the body is in motion. The perception of point-light biological motion activates posterior cortical areas of the brain. On the other hand, observation of others' actions is known to also evoke activity in motor and premotor areas in frontal cortex. In the present study, we investigated whether point-light biological motion animations would lead to activity in frontal cortex as well. We performed a human functional magnetic resonance imaging study on a high-field-strength magnet and used a number of methods to increase signal, as well as cortical surface-based analysis methods. Areas that responded selectively to point-light biological motion were found in lateral and inferior temporal cortex and in inferior frontal cortex. The robust responses we observed in frontal areas indicate that these stimuli can also recruit action observation networks, although they are very simplified and characterize actions by motion cues alone. The finding that even point-light animations evoke activity in frontal regions suggests that the motor system of the observer may be recruited to "fill in" these simplified displays.
\end{abstract}

Key words: biological motion; premotor cortex; functional MRI; action observation; motion; frontal

\section{Introduction}

The perception of other individuals' movements and actions is important for tracking and hunting prey, detecting and avoiding predators, and, in many species, social interaction. In humans and at least some other primates, premotor areas are involved in the perception of others' actions. Recent research has shown that there are "mirror neurons" in the macaque frontal cortex in area F5 that fire during both action production and action perception (Gallese et al., 1996; Rizzolatti et al., 1996a, 2001; Ferrari et al., 2003). Studies on humans have also demonstrated the involvement of motor and premotor areas in action observation, indicating that humans may use information from their own body representations in understanding the actions of others (Fadiga et al., 1995; Grafton et al., 1996; Rizzolatti et al., 1996b; Decety et al., 1997; Iacoboni et al., 1999; Buccino et al., 2001; Grèzes et al., 2003).

Besides the visual perception of actions, other components of actions also drive neurons in premotor areas. Auditory mirror neurons respond to the sound of actions (such as the sound of a peanut cracking) (Kohler et al., 2002), and "canonical neurons"

Received Jan. 3, 2004; revised May 18, 2004; accepted May 24, 2004.

This research was supported by National Science Foundation Behavioral and Cognitive Sciences Grant 0224321 (M.I.S.) and National Institutes of Health Grant R01 DC00216 (E.B.). We thank R. Buxton, L. Frank, T. Liu, and E. Wong from the University of California San Diego Center for fMRI for their support and pulse sequence, hardware development, and software development; E. Grossman for sharing stimuli; and M. lacoboni, F. Dick, and the anonymous reviewers for their comments.

${ }^{\dagger}$ Deceased, Dec. 13, 2003.

Correspondence should be addressed to Ayse Pinar Saygin, Department of Cognitive Science, University of California, San Diego, 9500 Gilman Drive, La Jolla, CA 92093-0515. E-mail: asaygin@cogsci.ucsd.edu.

DOI:10.1523/JNEUROSCI.0504-04.2004

Copyright $\odot 2004$ Society for Neuroscience $\quad 0270-6474 / 04 / 246181-08 \$ 15.00 / 0$ respond to the target objects of actions (such as a visually presented peanut) (Murata et al., 1997). The present study investigates whether premotor areas can be driven solely by motion cues of actions. It is possible to define actions by motion cues alone using "point-light biological motion." Image sequences constructed from point-lights attached to the limbs of a human actor can readily be identified as depicting actions, although they do not define a form when stationary (Johansson, 1973). These animations convey surprisingly detailed information about movements of the human body, despite using motion signals almost exclusively and lacking other visual cues such as color, shading, and contours. Given that point-light biological motion figures depict actions, could their perception also recruit frontal cortex? Or are these stimuli too simplified to drive the neural activity in frontal action observation areas?

Previous neurophysiological and neuroimaging studies of point-light biological motion perception have not typically reported activations in frontal regions. Instead, areas identified in these studies include the superior temporal gyrus and superior temporal sulcus (STS) (Grossman et al., 2000; Grèzes et al., 2001; Vaina et al., 2001; Beauchamp et al., 2003; Puce and Perrett, 2003), the motion-sensitive region MT (middle temporal visual area) and surrounding areas (MT+) (Grèzes et al., 2001; Vaina et al., 2001), the parietal cortex (Bonda et al., 1996; Grèzes et al., 2001; Vaina et al., 2001), and other regions in visual cortex (Vaina et al., 2001; Servos et al., 2002).

In the present study, using functional magnetic resonance imaging ( $\mathrm{fMRI}$ ), we investigated whether frontal action observation areas are involved in the perception of whole-body biological motion. Our approach was to use a relatively standard paradigm to identify regions in the brain that are responsive to biological 


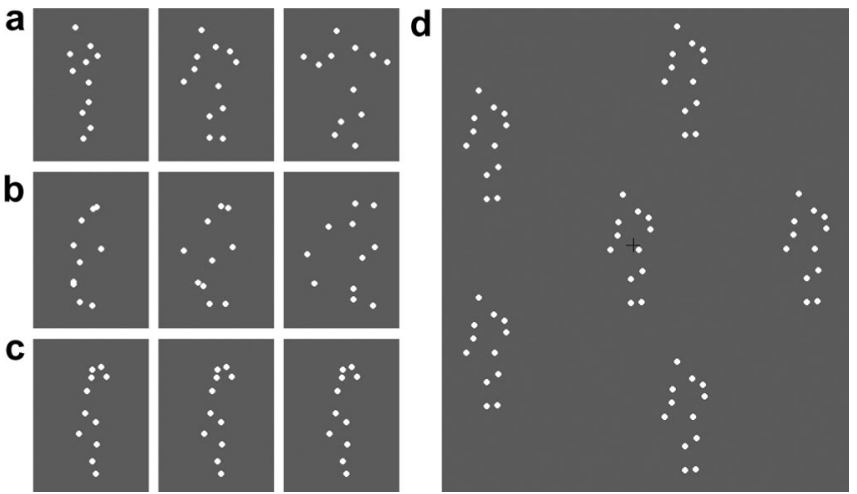

Figure 1. Example frames for the three stimulus conditions. Three (of 20) frames are shown from one animation each for biological motion ( $a$ ), scrambled biological motion $(b)$, and static point-lights ( $c$ ) (baseline) conditions in the experiment. The biological motion animation in this example depicts frames from an actor throwing an object (e.g., a ball). The static point-lights condition does not have any motion, and hence all frames are the same. In $d$, an example screenshot from the actual experiment (biological motion condition) is shown. All six copies of the figure executed the same motion.

motion. However, we used a combination of methods in our experimental design, fMRI acquisition, image processing, and data analysis to maximize signal in frontal cortex.

\section{Materials and Methods}

Participants. Twelve participants with no known visual or neurological abnormalities (seven females, aged 22-34) participated in this study. Eleven participants were unaware of the main hypothesis of the study, and one participant was an author. Subjects gave informed consent, according to procedures approved by the Institutional Review Board of the University of California.

Experimental design and procedure. Participants were scanned as they viewed point-light biological motion animations, scrambled versions of the same animations, and stationary point-light figures. Scrambled animations, which contain the same local motion cues but not the form defined by biological motion, have been used as control stimuli in some previous studies of biological motion processing (Grossman et al., 2000; Servos et al., 2002). Because scrambled animations do not constitute actions, we would predict that an area that responds to the action information would respond significantly more to biological motion compared with the scrambled motion. We used a stationary point-light baseline condition so that activity during biological and scrambled motion could both be measured.

A blocked design was chosen to maximize statistical detection power (Liu et al., 2001), in which the blocks consisted of biological motion, scrambled biological motion, and baseline (stationary point-light images). Figure 1 depicts several individual frames from each of these kinds of stimuli. During the scan, the three block types were presented in pseudo-randomized order and lasted $24 \mathrm{sec}$ each. There were three runs, with 21 blocks in each run.

Point-light biological motion sequences were a subset of those used by Ahlstrom et al. (1997) and were created by videotaping an actor performing various activities and then encoding the joint positions in the digitized videos. Ten point-light actions were used in the present study, depicting walking, walking up stairs, jogging, jumping jacks, throwing, underarm throwing, skipping, stepping up, a high kick into the air, and a lower kick. Six identical point-light figures were displayed at all times to maximize coverage of the visual field. The total area covered by the stimuli was $\sim 16-18^{\circ}$ of visual angle in diameter (Fig. $1 d$ ). The animations were presented at 20 frames/sec. Each animation was presented for $1 \mathrm{sec}$, with a delay of $250 \mathrm{msec}$ between animations and an extra $250 \mathrm{msec}$ interval between blocks. The joints of each point-light actor were represented by 12 small dots, each subtending $\sim 17$ arcmin of visual angle against a uniform dark background. For all point-light animations, the visual spatial locations stimulated were maintained approximately the same. To achieve this, for the biological motion animations, when the action depicted motion that would normally result in the figure moving in space (e.g., walking), the point-light figure was adjusted such that the figure did not leave the region in which the animations were presented (e.g., the figure walked in place, as if on a treadmill). There was a small, dark red cross hair at the center of the visual field to help subjects maintain central fixation and to minimize eye movements.

Scrambled animations were created by randomizing the starting positions of the point-lights while keeping the trajectories intact, except that each point-light could be randomly rotated in $90^{\circ}$ increments and/or mirror inverted. The rotation and mirror inversion of dots during scrambling additionally disrupts local form information that may remain after spatial scrambling. The starting positions were chosen randomly within a region such that the total area encompassed by the figure was similar to that of the real figures. Ten scrambled animations and 10 static frames were used.

The experiment was programmed and run using Matlab (MathWorks, Natick, MA) and the Psychophysics Toolbox (Brainard, 1997; Pelli, 1997). Stimuli were projected onto a screen that was suspended above the subject's torso, using an XGA video projector through a custom lens (Buhl Optical, Rochester, NY), and were viewed through a mirror that was placed inside the head coil. We used an adjustable bite bar to minimize head movements during the scan.

To control for differences in attention across conditions as much as possible, subjects were asked to perform a simple task of judging whether the color of the point-lights in each trial were green or not; the task was the same regardless of stimulus type. Responses were collected with a Lumitouch button box (Photon Control, Burnaby, Canada).

Pilot data were acquired from individual subjects using alternate tasks or with no task before the present design was finalized; activation patterns observed in these pilot scans resembled those found in the results of the analyses reported here (see below). However, during passive observation scans, pilot subjects often reported feeling inattentive, so we used the color-monitoring task to keep subjects alert. The point-lights were presented only in white, green, or yellow, and the task was "green or not." The green and yellow colors were similar enough that sustained attention was required to avoid false alarms. This task was chosen because performance does not depend on the form of different visual stimuli, so the subject's attention is focused on a feature of the stimulus (color) that can be varied in the same manner across the three conditions (biological, scrambled, and static). Finally, the task does not vary in difficulty across the different types of stimuli (confirmed in behavioral data, with accuracy in the task as follows: biological motion, $98.2 \%$; scrambled motion, 98.4\%; static point-lights, 97.8\%) ( $p>0.05$ for all comparisons).

Many visual fMRI studies use one-back working memory tasks to engage subjects' attention, which means that subjects monitor for repetitions of the visual stimuli as they are presented (Kanwisher et al., 1997). However, our pilot investigations and post-study subject interviews revealed that this task may not be ideal here because the difficulty of the working memory task varies by condition. To measure this more precisely, we asked 12 subjects to perform a one-back working memory task with our three stimulus types outside the scanner. The results confirmed that indeed the one-back task varies in difficulty for these stimuli. Accuracy was found to vary significantly by condition as follows: biological motion, $91.9 \%$; scrambled motion, $87.0 \%$; and static point lights, $96.1 \%$ ( $p<0.05$ for all comparisons). Compared with biological motion, the task is harder with scrambled motion as a result of the unfamiliarity of the stimuli and easier with static point-lights because the final and initial frames of successive matching stimuli are identical. Because working memory tasks often activate frontal areas (Smith and Jonides, 1999), such variation in task difficulty across conditions would complicate the interpretation of activity in frontal action observation areas.

Image acquisition. Scanning was performed on a 4 tesla Varian (Palo Alto, CA) scanner, equipped with a TEM (transverse electromagnetic resonator) transmit/receive head coil (Nova Medical, Wakefield, MA), at the University of California San Diego Center for fMRI (La Jolla, CA). We acquired three runs of functional data ( $509 \mathrm{sec}$ each) using a wholehead echo-planar imaging (EPI) sequence [repetition time (TR), 2400 msec; echo time (TE), $26.3 \mathrm{msec}$; flip angle, $90^{\circ}$; 32 axial slices with 
interleaved acquisition; in-plane resolution of $3.75 \times 3.75 \mathrm{~mm}$; and through-plane resolution of $3.8 \mathrm{~mm}$ with $0 \mathrm{~mm}$ gap]. Experimental stimuli began after three TRs to allow the magnetization to reach steady state.

Given that this study was performed on a high-field-strength magnet, magnetic susceptibility-induced artifacts were a significant concern. To help minimize these, we used a careful manual shimming routine and adjusted both linear $(n=3)$ and higher-order $(n=5)$ shims. In addition, a per-voxel equilibrium longitudinal magnetization (B0) field map (estimated from a set of multiecho EPI images) was at the beginning of each scan session, after shimming, and was used to estimate the residual nonflatness of the $\mathrm{B} 0$ field. These data were then used to correct for magnetic field inhomogeneities, which cause displacements in the phase-encode direction (Reber et al., 1998).

After functional scanning, a single structural volume for each subject was acquired using a T1-weighted magnetization-prepared rapid gradient echo (MPRAGE) sequence (TR, $10.5 \mathrm{msec}$; TE, $4.8 \mathrm{msec}$; flip angle, $11^{\circ} ; 1 \times 1 \times 1.5 \mathrm{~mm}$ voxels). This structural scan was used as an intermediate step in spatially aligning the functional images to highresolution $(1 \times 1 \times 1 \mathrm{~mm})$ T1-weighted MPRAGE scans previously obtained on a 3 tesla Varian scanner or a 1.5 tesla Siemens Vision (Erlangen, Germany) clinical scanner. These previously obtained highresolution scans were used to reconstruct the cortical surface of each subject, as described previously (Dale et al., 1999; Fischl et al., 1999a).

Image processing and analysis. Image preprocessing and statistical analysis were performed using Analysis of Functional Neuroimages (AFNI) (Cox, 1996), FreeSurfer (Dale et al., 1999; Fischl et al., 1999a), and Matlab (MathWorks, Natick, MA) software packages.

For each individual subject, the B0 field maps were used to correct for distortions in the phase-encode direction using in-house software developed at the University of California San Diego fMRI center by L. Frank. The three runs were concatenated (yielding 630 volumes) and spatially registered in three-dimensional space for motion correction using AFNI programs. Estimates of the three translation and three rotation parameters were computed during this registration and saved. The AFNI program $3 \mathrm{dDeconvolve} \mathrm{was} \mathrm{used} \mathrm{to} \mathrm{fit} \mathrm{a} \mathrm{general} \mathrm{linear} \mathrm{model} \mathrm{at} \mathrm{each} \mathrm{voxel.}$ The model contained four parameters for each of the two nonbaseline conditions, modeling hemodynamic responses at different lag times (0-3 TRs), three parameters for each run to account for slow drifts, and the six motion vectors as determined during motion correction. For individual subject analyses, the contrast between the two conditions (biological vs scrambled motion) was performed by using $F$ tests to compare the sums of the four parameters (i.e., the areas under the fitted hemodynamic response functions).

The group data were analyzed using cortical surface-based methods (Dale et al., 1999; Fischl et al., 1999a,b). Each subject's cortical surface was reconstructed and was then morphed to an average spherical representation of the cerebral hemispheres that optimally aligns the sulcal and gyral features across subjects, through a procedure that aims to match these features across subjects while minimizing metric distortion (Fischl et al., 1999b). To perform functional analysis on the sphere, each subject's volume-based individual statistical maps of coefficients were first interpolated onto the spherical representation of their hemispheres using FreeSurfer. Then these maps were morphed and resampled into the common spherical space. At this stage, 50 steps of spatial smoothing on the spherical surface were applied. We performed simulations with a set of surfaces and a set of points on the cortex and found this to correspond approximately to a Gaussian filter with a full-width at half-maximum of $7 \mathrm{~mm}$, along the cortical surface (A. P. Saygin and D. J. Hagler, unpublished simulations). A two-factor ANOVA was performed on the spherical surface using a mixed-effects model, with condition as the fixed effect and subjects as the random effect. The resulting statistics were then transferred onto the inflated cortical surface of a single subject for display.

To examine responses to biological motion and scrambled biological motion more closely, we also defined regions of interest (ROIs) and examined the responses in these areas. We selected inferior frontal (IF) and premotor (Prem) cortical regions as our main regions of interest on the basis of previously known involvement of these areas in action observation. We also studied the posterior superior temporal sulcal (pSTS) region because it is an area known to respond to point-light biological motion. These ROIs were drawn on the cortical surface of each hemisphere of each individual subject using FreeSurfer and saved as surface patches. Anatomical criteria were as follows. The IF ROI contained the inferior frontal gyrus (IFG) and the inferior frontal sulcus (IFS) and was bounded by (but did not contain any cortex from) the middle frontal gyrus, precentral sulcus, lateral orbital sulcus, and the Sylvian fissure. The Prem ROI was drawn on the lateral cortical surface and consisted of the precentral gyrus and the posterior bank of the precentral sulcus but did not extend into the middle and superior frontal gyri or the central or inferior frontal sulci. Although most action observation studies have observed responses in ventral portions of premotor cortex, it is also known that responses in premotor cortex during observation of body actions may be somatotopically specific (Buccino et al., 2001). Because our stimuli contain actions of the whole body, we found it appropriate to include the whole lateral extent of the precentral region to cover a large extent of human premotor cortex rather than only the more ventral portions that correspond mostly to the arm and hand representations. This ROI did not extend into the medial surface of the precentral gyrus. Finally, the pSTS ROI was drawn to include the posterior half of the superior temporal sulcal cortex.

For these three anatomical ROIs, time courses were extracted based on voxels that were responsive to motion, either biological or scrambled, at $p<10^{-3}$. Because the design of the experiment was a mixed-block design (to maximize signal), biological motion and scrambled motion blocks could follow each other, and thus the hemodynamic responses to each kind of stimulus could overlap. Thus, to extract the blood oxygen level-dependent (BOLD) responses corresponding to each condition in our experiment, we used the AFNI program 3dDeconvolve. The mean time course from each ROI of each hemisphere was averaged and deconvolved with a model containing 16 parameters each for the biological motion and the scrambled motion conditions, corresponding to stimulus time points throughout the experiment (10 stimulus TRs per block, the two TRs preceding each block and the four TRs after each block). The extracted BOLD responses for biological motion and scrambled biological motion across the 16 time points were then averaged across subjects for each ROI and hemisphere, resulting in average estimated BOLD responses for biological motion and scrambled biological motion blocks.

\section{Results}

The group results for the 12 subjects are depicted in Figure 2. We first discuss responses to biological motion and scrambled biological motion compared with the static baseline (Fig. $2 a, b$ ) before moving to the main comparison of interest, which is the contrast between biological motion and scrambled motion (Fig. $2 c$ ). The activations against baseline are important because they illustrate the areas that respond to both biological and scrambled motion, which cannot be inferred from a difference image.

When biological motion observation was compared with the static point-light observation baseline (Fig. $2 a$ ), we found a robustly responsive region along the inferior frontal and precentral sulci bilaterally, indicating that point-light animations indeed recruit frontal areas known to be involved in action observation. This activation followed inferior frontal and precentral sulci in a fairly continuous manner, but Talairach coordinates of the most significantly responsive points in the inferior frontal, inferior precentral, and superior precentral sulci are reported online in supplemental Table 1 (available at www.jneurosci.org).

In posterior brain regions, compared with the static baseline condition, biological motion led to extensive activation in occipital, temporal, and parietal cortex, extending along both the ventral and dorsal visual streams. Because many of these regions were also responsive to scrambled motion (see below), motion processing may account for much of this activity. The peak of this continuous response was in the lateral temporal cortex, inferior to the STS, near anatomical areas that are known to respond strongly to motion stimuli (human MT, medial superior tempo- 
ral area MST, and surrounding regions; henceforth $\mathrm{MT}+)$. Peak coordinates here and in the pSTS, intraparietal sulcus, inferotemporal cortex, and the posterior insular cortex (which has been considered the putative human analog of the monkey parietoinsular vestibular cortex, or PIVC) (for review, see Güldin and Grusser, 1998) are reported in supplemental Table 1 (available at www.jneurosci.org).

Scrambled biological motion, relative to the static point-light baseline, activated many of the same regions as biological motion in occipital, temporal, parietal, and posterior insular cortex, although the activation was noticeably less extensive (Fig. 2b) (for coordinates of activation peaks, see supplemental Table 1, available at www.jneurosci.org). The most significant responses were once again in posterior lateral temporal cortex around $\mathrm{MT}+$, reflecting motion processing. On the other hand, scrambled biological motion did not evoke much activation in frontal cortex, even when compared with baseline and even at low thresholds. Indeed, no difference was visible between scrambled motion and the static baseline in the left hemisphere (LH). In the right hemisphere $(\mathrm{RH})$, a small area of activation in the precentral sulcus associated with scrambled motion against baseline was found, but this was weaker and less extensive than the activation seen for biological motion.

When biological motion and scrambled biological motion responses were compared directly, we found that a region in the left IFS, at its junction with and par-

tially extending into the precentral sulcus, responded significantly more to biological motion (Fig. $3 c$ ). In fact, this was the most significantly responsive area for this contrast in the whole brain [peak Talairach coordinates $(-41,14,18)$ with $t=9.8$ ]. There were less significant peaks in the inferior precentral sulci bilaterally [left hemisphere peak at $(-37,5,25)$ with $t=5.5$ and right hemisphere peak at $(34,7,27)$ with $t=5.2]$. Thus, we found support for the hypothesis that motion information in body actions can drive neural activity in frontal cortical regions.

In line with previous work, we also found lateral temporal regions that responded more strongly to biological motion than to scrambled motion. Although the peak voxels were in rather similar locations in the two hemispheres (see supplemental Table 1 , available at www.jneurosci.org), the region that was significantly responsive to the contrast extended more anteriorly and superiorly toward the STS in the left hemisphere, and, although these areas were responsive in the right hemisphere as well, the strongest responses lay more posteriorly in this hemisphere. Finally, a region in left ventrolateral inferotemporal cortex (most anterior activation in temporal cortex seen in Fig. $2 c$ ) also showed significant responses to biological motion compared with scrambled biological motion. We did not find brain areas that preferred scrambled motion over biological motion.

Note that the large activated regions in temporal cortex likely contain multiple functional visual areas because they are very close to or partially overlapping with areas that have been reported in previous studies to be responsive to simple motion (Tootell et al., 1995), visual form of objects (Grill-Spector et al., 1999), human bodies (Downing et al., 2001), and shape-frommotion (Murray et al., 2003). In fact, we verified this by examination of several individual subjects' biological motionresponsive regions identified in this study in relation to results from localizer scans performed in our laboratory and found that, at the individual subject level, brain areas that have a preference for biological motion have considerable overlap with areas that respond to simple motion, object form, human faces, and, especially, human body form (data not shown). Additionally, although a large area in lateral temporal is cortex responsive to biological motion, it has also been observed that different portions of human temporal cortex have relative preferences for different kinds of motion stimuli, such as biological versus artifact motions (Beauchamp et al., 2003; Pelphrey et al., 2003).

We next examined the average hemodynamic responses to the biological motion and scrambled biological motion blocks across the 12 subjects for two anatomical regions in frontal cortex that are known to respond during action observation: IF and Prem cortex. We also studied the pSTS because it is known to respond more to biological motion than to scrambled biological motion (for anatomical boundaries of these ROIs, see Materials and 


\section{a. Inferior Fronta}
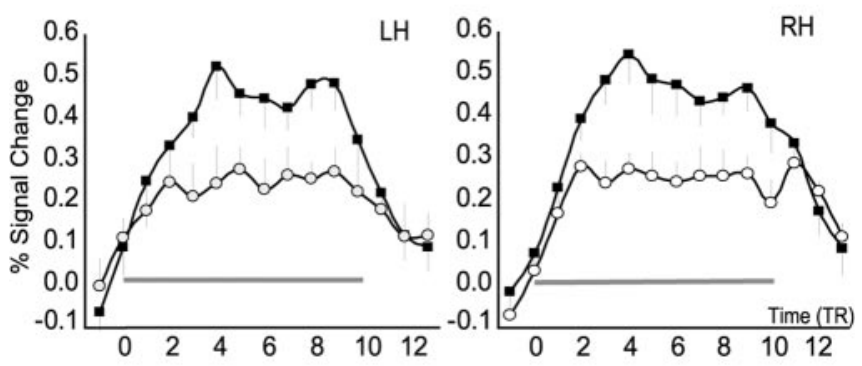

\section{b. Premotor}
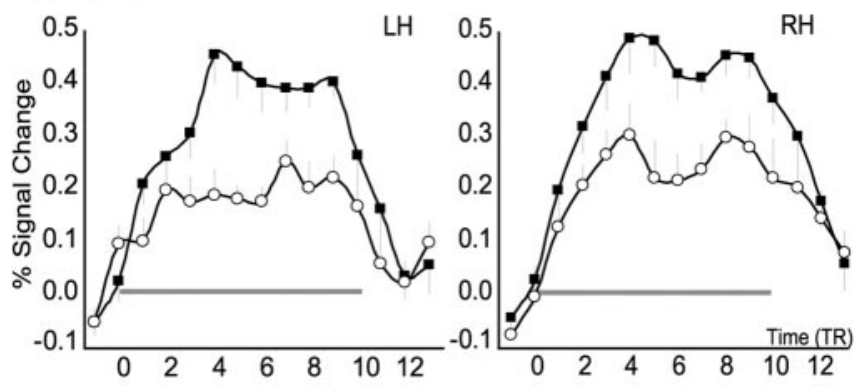

\section{c. Posterior STS}

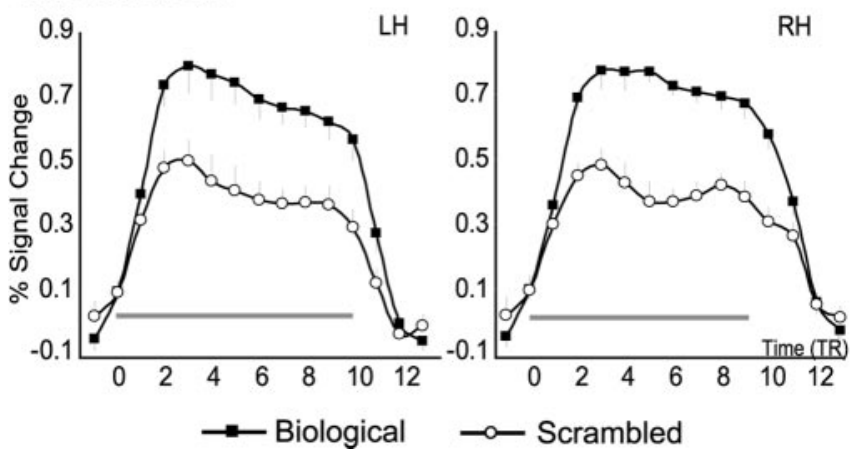

Figure 3. Percent MR signal change across time for the biological motion and scrambled biological motion blocks in the IF, Prem, and pSTS regions of interest. The filled squares depict the signal for biological motion, and the open circles depict the signal for scrambled motion. The error bars show SE across the 12 subjects. In IF, the mean number of voxels included in the ROI analyses across the 12 subjects was 53 in LH, 58 in RH; in Prem, the mean number was 64 in LH, 80 in RH; and in pSTS, the mean number was 108 in LH, 98 in RH. The horizontal gray line marks the actual duration of stimuli ( 10 TRs or $24 \mathrm{sec}$ ).

Methods). Figure 3 depicts the percent signal change for each of these ROIs in each of the two hemispheres.

In contrast to most previous studies, the addition of a baseline condition in our experiment (stationary point-light observation while executing the color-monitoring task) allowed us to examine responses to both biological and scrambled motion. In all ROIs in both hemispheres, responses to biological motion were much larger than the responses to scrambled motion, although scrambled motion can also be seen to give rise to responses significantly above baseline in all regions. The amplitude of the signal change in pSTS was greatest, which is not unexpected because this is a posterior brain area known to be involved in the visual perception of biological motion. Signal change in pSTS for scrambled motion was also quite high, but the area showed a stronger response to the biological motion stimuli, as has been previously observed (Grossman et al., 2000). Responses in frontal cortex were also strong. We found very similar response patterns to those in pSTS in both the IF and the Prem ROIs; the percent signal change in these regions for biological motion was much greater than that for scrambled biological motion. Moreover, the difference in the responses to the two stimulus types in frontal cortex was similar in magnitude to the difference observed in the pSTS. To quantify this, we calculated the area under the estimated hemodynamic response curves for the biological and scrambled motion conditions, and we found that the size of the response in the scrambled motion condition as a fraction of the response in the biological motion condition was very similar across ROIs: IF, 56.3\%; Prem, 55.7\%; and pSTS, 58.6\%. This suggests that the frontal regions are just as selective for biological motion as the pSTS.

As with most fMRI studies, group analyses show the strongest and most reliable responses to biological motion across a group of subjects, whereas for individual subjects there is some variability in the activation patterns obtained. In Figure 4, we show biological versus scrambled motion contrasts for three individual subjects. There were some subjects who showed significant responses to biological motion compared with scrambled biological motion in parietal cortex (e.g., subjects 2 and 3), consistent with some previous results [Bonda et al., 1996 (only for hand actions); Grèzes et al., 2001; Vaina et al., 2001]. In some individual subjects, the response extended ventrally toward inferotemporal cortex (e.g., subject 3 and in the left hemisphere of subject 2; the extension is partially visible in the lateral view; ventral view not shown), which has also been reported in some previous studies (Vaina et al., 2001; Grossman and Blake, 2002). The frontal response, which is the focus of this study, also showed some variability. Most notably, several subjects' frontal activation extended dorsally along the precentral sulcus, beyond the IFS focus, which emerged from the group average as the most responsive region to biological motion (subjects 1,2 , and 3 ). Other subjects had activation in slightly more anterior or inferior regions of the IFS (subject 2 and a smaller focus seen in the left hemisphere of subject 1). For some subjects, the response in the posterior insula (or human PIVC) showed a significant difference between biological and scrambled motion (e.g., seen bilaterally in subjects 2 and 3 ).

Finally, overlaid on the activation maps for subject 3 are the areas activated in a separate scanning session for biological versus scrambled motion, in which this subject performed a one-back working memory task instead of the color-monitoring task. As noted above, behavioral data indicate that the one-back task is more difficult for scrambled motion, presumably because the items to be compared are unfamiliar. However, the areas activated were very similar across the two tasks; in particular, the IFS and premotor cortex responded significantly more strongly to biological motion during the one-back task. Also shown is average percent signal change in each of the three ROIs (IF, Prem, and pSTS, right and left hemispheres averaged) for each task. In each ROI, the response pattern (biological motion $>$ scrambled motion) was the same regardless of the task. These results suggest that the activated frontal areas are unlikely to reflect general attentional differences between the conditions because the oneback task is more challenging for the scrambled condition and hence presumably places greater demands on working memory, executive systems, and attentional systems, yet even in this case the IFS and premotor regions respond more strongly to biological motion.

\section{Discussion}

This study aimed to investigate whether frontal areas known to be activated by action observation would also respond to actions characterized solely by motion cues. We used point-light biolog- 


\section{a. Subject 1}
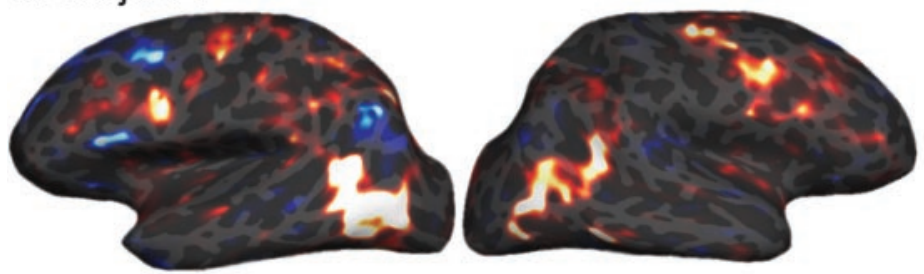

\section{b. Subject 2}
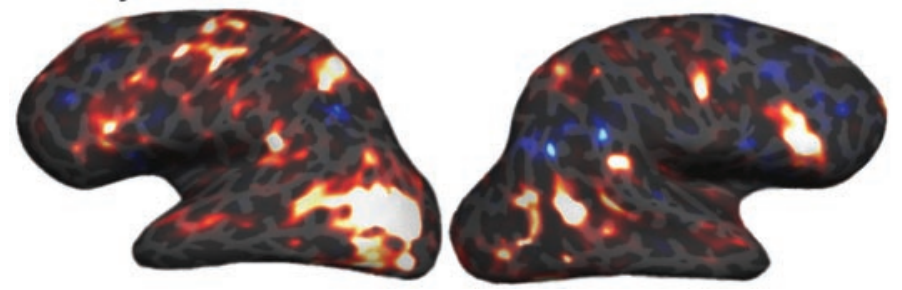

\section{c. Subject 3}
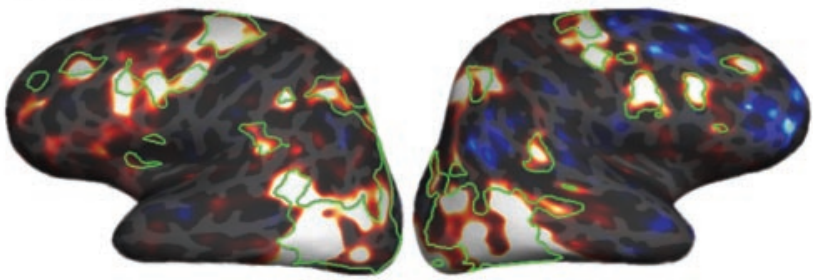

Subject 3

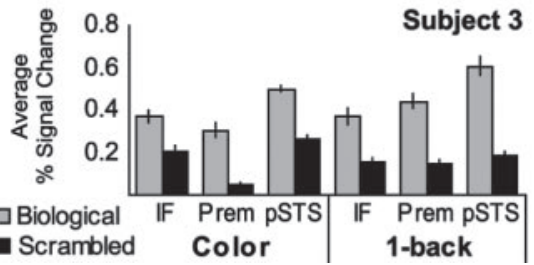

Figure 4. Example individual subject results. Results for the biological motion versus scrambled biological motion contrast is shown on inflated lateral views of three subjects' hemispheres. The color bar shows the colors in the images, and the discrete swatches mark colors that correspond to $p$ values smaller than $10^{-3}, 5 \times 10^{-4}, 10^{-4}$, and $10^{-5}$, or $t>3.3, t>3.5, t>3.9$, and $t>4.5$, respectively. In $a$, subject 1 can be seen to show a pattern similar to the frontal and temporal response pattern found in the group study, with more extension into precentral sulcus. In $b$ and $c$, subject 2 and subject 3 are depicted showing strong activity in inferior frontal and premotor areas in frontal cortex, in addition to superior temporal, parietal, posterior insular, and inferotemporal cortex (left-lateralized for subject 2; bilateral for subject 3 with extension into ventral cortex, which is not visible). In c, additional data for subject 3 is shown from a separate scan in which the same stimuli were presented in a one-back working-memory task with different attentional requirements. To show precise alignment of activated regions in the two different tasks, regions responsive to the biological motion versus scrambled biological motion contrast during the one-back task at $p<10^{-3}$ are outlined in green and superimposed onto the surface on which the data from the main experiment (color-monitoring task) was rendered. Also shown in c is a graph of the average percent signal change in our three ROls (IF, Prem, pSTS; data from left and right hemispheres combined) for these two scanning conditions, which revealed that all of these areas were more responsive to biological motion than scrambled motion under both task conditions.

ical motion animations of whole-body actions that have consistently activated superior temporal cortical areas in most previous human neuroimaging studies (Bonda et al., 1996; Grossman et al., 2000; Grèzes et al., 2001; Vaina et al., 2001; Beauchamp et al., 2003) (but see Servos et al., 2002). Our approach was to keep the experimental design straightforward (block design with two motion conditions and one static baseline, using a simple task) but to use a combination of methods to increase the signal from frontal cortex.

We found that frontal cortex showed a robust response to point-light biological motion. Compared with static point-lights, there was activation that followed the precentral and inferior frontal sulci bilaterally. Frontal areas also showed selective responsivity to biological motion compared with scrambled biological motion. These results support the view that perception of the motion information in body actions can drive inferior frontal and premotor areas involved in action perception.

When we investigated the MR signal in IF, Prem, and pSTS ROIs, we saw that the BOLD response in frontal areas showed a very similar pattern to that in pSTS, an area whose importance in biological motion processing is already established. More precisely, IF and Prem were as selective as pSTS to the contrast between biological and scrambled motion, as revealed by both the surface-based group analysis and the ROI analyses.

Frontal cortical areas (as well as sensory areas in other parts of the brain) are known to be modulated by attention (for review, see Pessoa et al., 2003). However, the frontal areas observed in our study are unlikely to primarily reflect differences in attention across the conditions. A one-back working-memory task, which is more attentionally demanding for the scrambled condition, revealed the same pattern of responses (biological $>$ scrambled) in the inferior frontal and precentral areas activated with the "neutral" color-monitoring task. Another consideration is that the areas activated in this study overlap only partially with areas thought to be important for attentional control. The very dorsal extent of the premotor activity we observed likely overlaps with the location of the frontal eye fields near the junction of the superior frontal sulcus and superior precentral sulcus (Paus, 1996), and attention shifts have also been reported to activate an area in the inferior precentral sulcus (Beauchamp et al., 2001). However, the IFS, in which we saw the largest differences between biological and scrambled motion in the surface-based group analysis, is not thought to be involved in these spatial attentional processes.

The present study is the first study that shows a clear response to point-light biological motion animations in frontal areas known to be involved in action observation. There are a few related results from previous studies. First, we found inferior frontal lesion sites (as well as superior temporal and parietal sites) to be implicated in biological motion perception deficits in a group of unilateral stroke patients (A. P. Saygin and S. M. Wilson, unpublished observations). Second, right lateralized frontal activation in Brodmann area (BA) 47 and extending into BA 45 was found in a previous fMRI study of biological motion processing (Vaina et al., 2001). However, in that study, subjects were viewing both biological and scrambled biological motion stimuli within a single condition and performing a discrimination task between the two kinds of motion, which makes the interpretation of this activation difficult. Santi et al. (2003) also reported activation in BA 47 in the right hemisphere during biological motion perception. Note that BA 47 is inferior and anterior to regions typically active during action observation. In the same study, a large region of frontal activation, overlapping with known action observation networks in the left hemisphere, was found to be responsive to point-light biological motion. However, this area was responsive only to visible speech biological motion as subjects were trying to lip-read and did not respond during observation of whole-body biological motion actions. On the basis of these results, the authors suggested that the activation in these premotor and motor regions was linguistically specific. The present study, however, 
shows that body actions also evoke activity in these frontal regions. Perhaps the linguistic task they used (lip-reading) may have led to the relative differences they observed between speech and nonspeech biological motion observation.

In summary, although frontal cortical involvement has sometimes been observed in previous studies involving biological motion, no previous imaging study has shown responses specific to point-light biological motion actions in frontal areas known to be involved in action observation. Methodological differences between previous neuroimaging studies that examined biological motion perception and our experiment may account for the different results we obtained. First, we used a color-detection task as opposed to passive viewing or a working-memory task. Second, we presented multiple point-light animations at any given time. Finally, and probably most importantly, we took additional steps to maximize signal in our fMRI design, acquisition, and analysis methods (e.g., used a 4 tesla scanner, B0 field map correction, linear and higher-order shimming, and surface-based intersubject averaging methods).

Where do the frontal regions activated in our study lie in relation to areas identified in previous studies of action observation? Many action observation and imitation studies have pointed to the posterior IFG as being a particularly important area and a possible homolog for macaque area F5, which contains mirror neurons (Rizzolatti et al., 2001). Several action observation and imagery studies have found responses in premotor areas, as well (for review, see Jeannerod, 2001). We plotted on the cortical surface several reported peak activation coordinates from previously published studies that had action observation conditions and that found responses in inferior frontal cortex (Grafton et al., 1996; Rizzolatti et al., 1996b; Decety et al., 1997; Iacoboni et al., 1999; Grèzes et al., 2003). Several of these foci fell on the IFG, a few millimeters to a centimeter inferior to our activation (Grafton et al., 1996; Rizzolatti et al., 1996b; Iacoboni et al., 1999); one was a few millimeters anterior to our focus, again on the IFG (Decety et al., 1997); and one study reported a focus that is overlapping with our biological motion responses (Grèzes et al., 2003). However, because the reported peaks are points in the center of an activated region, they may still overlap with our responses.

Does this localization in the present study to the IFS rather than to the IFG have any significance? We suggest three possible reasons for the differences in precise localization. First, there are methodological differences between studies. The present study used surface-based group registration, which aims to optimally align particular sulci and gyri. The localization to the sulcus in the group results follows from the fact that the activation was generally localized to the sulcus for each individual subject. Second, the difference might depend on the fact that the actions in the present study were defined by motion alone, whereas previous action observation studies have used videotaped actions that contain many other visual cues such as form, contour, and color. It may be that slightly different frontal areas are engaged by different aspects of action perception. Third, and perhaps most likely, most previous studies have used hand-action stimuli (e.g., grasping), whereas in the present study whole-body actions were used to maintain contiguity with the previous literature on point-light biological motion processing. It has been shown that action observation activates premotor areas in a somatotopic manner (Buccino et al., 2001); therefore, it may be expected that actions involving different body parts would activate different regions. Because hand motor representations are ventral to representations for many body parts, such as the arms, shoulders, trunk, and legs (Preuss et al., 1996), the more superior focus that we observed could be attributable to the fact that our stimuli contained whole-body movements.

Finally, it is noteworthy that, in the macaque, mirror neurons in premotor cortex respond only to real actions performed in front of the monkey and not even to videotaped actions (Ferrari et al., 2003), whereas human premotor cortex responds even to point-light biological motion representing actions. This contrast between humans and macaques suggests that the human mirror neuron system may be more capable of processing abstract visual representations of actions.

Whereas others' actions are most often experienced through the visual system, an organism's own experience of performing the same action will involve motor, sensory, and proprioceptive representations (Barresi and Moore, 1996). A unified representation of action requires that perceived actions and performed actions be related to each other in the brain, although they are often experienced through different sensory modalities. In this context, the discovery that perception of actions can engage neural systems involved in production of actions has been an exciting development. The present study showed that human premotor cortex responds during the perception of actions defined by motion cues alone. Our findings suggest that we may be filling in these simplified animations using information from our own motor system, lending support to an analysis-by-synthesis view of action perception.

\section{References}

Ahlstrom V, Blake R, Ahlstrom U (1997) Perception of biological motion. Perception 26:1539-1548.

Barresi J, Moore C (1996) Intentional relations and social understanding. Behav Brain Sci 19:107-122.

Beauchamp MS, Petit L, Ellmore TM, Ingeholm J, Haxby JV (2001) A parametric fMRI study of overt and covert shifts of visuospatial attention. NeuroImage 14:310-321.

Beauchamp MS, Lee KE, Haxby JV, Martin A (2003) FMRI responses to video and point-light displays of moving humans and manipulable objects. J Cognit Neurosci 15:991-1001.

Bonda E, Petrides M, Ostry D, Evans A (1996) Specific involvement of human parietal systems and the amygdala in the perception of biological motion. J Neurosci 16:3737-3744.

Brainard DH (1997) The psychophysics toolbox. Spat Vis 10:433-436.

Buccino G, Binkofski F, Fink GR, Fadiga L, Fogassi L, Gallese V, Seitz RJ, Zilles K, Rizzolatti G, Freund HJ (2001) Action observation activates premotor and parietal areas in a somatotopic manner: an fMRI study. Eur J Neurosci 13:400-404.

Cox RW (1996) AFNI: software for analysis and visualization of functional magnetic resonance neuroimages. Comput Biomed Res 29:162-173.

Dale AM, Fischl B, Sereno MI (1999) Cortical surface-based analysis. I. Segmentation and surface reconstruction. NeuroImage 9:179-194.

Decety J, Grèzes J, Costes N, Perani D, Jeannerod M, Procyk E, Grassi F, Fazio F (1997) Brain activity during observation of actions: influence of action content and subject's strategy. Brain 120:1763-1777.

Downing PE, Jiang Y, Shuman M, Kanwisher N (2001) A cortical area selective for visual processing of the human body. Science 293:2470-2473.

Fadiga L, Fogassi L, Pavesi G, Rizzolatti G (1995) Motor facilitation during action observation: a magnetic stimulation study. J Neurophysiol 73:2608-2611.

Ferrari PF, Gallese V, Rizzolatti G, Fogassi L (2003) Mirror neurons responding to the observation of ingestive and communicative mouth actions in the monkey ventral premotor cortex. Eur J Neurosci 17:1703-1714.

Fischl B, Sereno MI, Dale AM (1999a) Cortical surface-based analysis. II. Inflation, flattening, and a surface-based coordinate system. NeuroImage 9:195-207.

Fischl B, Sereno MI, Tootell RB, Dale AM (1999b) High-resolution intersubject averaging and a coordinate system for the cortical surface. Hum Brain Mapp 8:272-284. 
Gallese V, Fadiga L, Fogassi L, Rizzolatti G (1996) Action recognition in the premotor cortex. Brain 119:593-609.

Grafton ST, Arbib MA, Fadiga L, Rizzolatti G (1996) Localization of grasp representations in humans by positron emission tomography. 2. Observation compared with imagination. Exp Brain Res 112:103-111.

Grèzes J, Fonlupt P, Bertenthal B, Delon-Martin C, Segebarth C, Decety J (2001) Does perception of biological motion rely on specific brain regions? NeuroImage 13:775-785.

Grèzes J, Armony JL, Rowe J, Passingham RE (2003) Activations related to "mirror" and "canonical" neurones in the human brain: an fMRI study. NeuroImage 18:928-937.

Grill-Spector K, Kushnir T, Edelman S, Avidan G, Itzchak Y, Malach R (1999) Differential processing of objects under various viewing conditions in the human lateral occipital complex. Neuron 24:187-203.

Grossman E, Donnelly M, Price R, Pickens D, Morgan V, Neighbor G, Blake R (2000) Brain areas involved in perception of biological motion. J Cognit Neurosci 12:711-720.

Grossman ED, Blake R (2002) Brain areas active during visual perception of biological motion. Neuron 35:1167-1175.

Güldin WO, Grusser OJ (1998) Is there a vestibular cortex? Trends Neurosci $21: 254-259$.

Iacoboni M, Woods RP, Brass M, Bekkering H, Mazziotta JC, Rizzolatti G (1999) Cortical mechanisms of human imitation. Science 286:2526-2528.

Jeannerod M (2001) Neural simulation of action: a unifying mechanism for motor cognition. NeuroImage 14:S103-S109.

Johansson G (1973) Visual perception of biological motion and a model for its analysis. Percept Psychophys 14:201-211.

Kanwisher N, McDermott J, Chun MM (1997) The fusiform face area: a module in human extrastriate cortex specialized for face perception. J Neurosci 17:4302-4311.

Kohler E, Keysers C, Umilta MA, Fogassi L, Gallese V, Rizzolatti G (2002) Hearing sounds, understanding actions: action representation in mirror neurons. Science 297:846-848.

Liu TT, Frank LR, Wong EC, Buxton RB (2001) Detection power, estimation efficiency, and predictability in event-related fMRI. NeuroImage 13:759-773.

Murata A, Fadiga L, Fogassi L, Gallese V, Raos V, Rizzolatti G (1997) Object representation in the ventral premotor cortex (area F5) of the monkey. J Neurophysiol 78:2226-2230.

Murray SO, Olshausen BA, Woods DL (2003) Processing shape, motion and three-dimensional shape-from-motion in the human cortex. Cereb Cortex 13:508-516.

Paus T (1996) Location and function of the human frontal eye-field: a selective review. Neuropsychologia 34:475-483.

Pelli DG (1997) The video toolbox software for visual psychophysics: transforming numbers into movies. Spat Vis 10:437-442.

Pelphrey KA, Mitchell TV, McKeown MJ, Goldstein J, Allison T, McCarthy G (2003) Brain activity evoked by the perception of human walking: controlling for meaningful coherent motion. J Neurosci 23:6819-6825.

Pessoa L, Kastner S, Ungerleider LG (2003) Neuroimaging studies of attention: from modulation of sensory processing to top-down control. J Neurosci 23:3990-3998.

Preuss TM, Stepniewska I, Kaas JH (1996) Movement representation in the dorsal and ventral premotor areas of owl monkeys: a microstimulation study. J Comp Neurol 371:649-676.

Puce A, Perrett D (2003) Electrophysiology and brain imaging of biological motion. Philos Trans R Soc Lond B Biol Sci 358:435-445.

Reber PJ, Wong EC, Buxton RB, Frank LR (1998) Correction of off resonance-related distortion in echo-planar imaging using EPI-based field maps. Magn Reson Med 39:328-330.

Rizzolatti G, Fadiga L, Gallese V, Fogassi L (1996a) Premotor cortex and the recognition of motor actions. Brain Res Cogn Brain Res 3:131-141.

Rizzolatti G, Fadiga L, Matelli M, Bettinardi V, Paulesu E, Perani D, Fazio F (1996b) Localization of grasp representations in humans by PET. 1. Observation versus execution. Exp Brain Res 111:246-252.

Rizzolatti G, Fogassi L, Gallese V (2001) Neurophysiological mechanisms underlying the understanding and imitation of action. Nat Rev Neurosci 2:661-670.

Santi A, Servos P, Vatikiotis-Bateson E, Kuratate T, Munhall K (2003) Perceiving biological motion: dissociating visible speech from walking. J Cognit Neurosci 15:800-809.

Servos P, Osu R, Santi A, Kawato M (2002) The neural substrates of biological motion perception: an fMRI study. Cereb Cortex 12:772-782.

Smith EE, Jonides J (1999) Storage and executive processes in the frontal lobes. Science 283:1657-1661.

Tootell RB, Reppas JB, Kwong KK, Malach R, Born RT, Brady TJ, Rosen BR, Belliveau JW (1995) Functional analysis of human MT and related visual cortical areas using magnetic resonance imaging. J Neurosci 15:3215-3230.

Vaina LM, Solomon J, Chowdhury S, Sinha P, Belliveau JW (2001) Functional neuroanatomy of biological motion perception in humans. Proc Natl Acad Sci USA 98:11656-11661. 